\title{
Spatial imaging of the spin Hall effect and current-induced polarization in two-dimensional electron gases
}

\author{
V. SIH, R. C. MYERS, Y. K. KATO, W. H. LAU, A. C. GOSSARD AND D. D. AWSCHALOM* \\ Center for Spintronics and Quantum Computation, University of California, Santa Barbara, California 93106, USA \\ *e-mail: awsch@physics.ucsb.edu
}

(5) pin-orbit coupling in semiconductors relates the spin of an electron to its momentum, and provides a pathway for electrically initializing and manipulating electron spins for applications in spintronics ${ }^{1}$ and spin-based quantum information processing ${ }^{2}$. This coupling can be regulated with quantum confinement in semiconductor heterostructures through band-structure engineering. Here we investigate the spin Hall effect ${ }^{3,4}$ and current-induced spin polarization ${ }^{5,6}$ in a two-dimensional electron gas confined in (110) AlGaAs quantum wells using Kerr rotation microscopy. In contrast to previous measurements ${ }^{7-10}$, the spin Hall profile shows complex structure and the current-induced spin polarization is out-of-plane. The experiments map the strong dependence of the current-induced spin polarization to the crystal axis along which the electric field is applied, reflecting the anisotropy of the spin-orbit interaction. These results reveal opportunities for tuning a spin source using quantum confinement and device engineering in non-magnetic materials.

Previous measurements in bulk epilayers of $n-G a A s$ and $\mathrm{n}$-InGaAs (ref. 7) and in a two-dimensional hole gas ${ }^{8}$ provide experimental evidence for the spin Hall effect ${ }^{3,4,11,12}$, but it remains unclear whether the dominant mechanism is extrinsic or intrinsic. The extrinsic mechanism ${ }^{3,4}$ is mediated by spin-dependent scattering, where spin-orbit coupling mixes the spin and momentum eigenstates. Alternatively, an intrinsic spin Hall mechanism has been proposed ${ }^{11,12}$ that is an effect of the momentum-dependent internal magnetic field $B_{\text {int }}$. This internal field arises from spin-orbit coupling, which introduces a spin splitting for electrons with non-zero wavevector $\mathbf{k}$ in semiconductors lacking an inversion centre. For example, bulk inversion asymmetry exists owing to the zincblende crystal structure of GaAs and introduces the Dresselhaus spin splitting ${ }^{13}$, whereas structural inversion asymmetry is present in heterostructures that are not symmetric along the growth direction and leads to an in-plane spin splitting known as the Bychkov-Rashba effect ${ }^{14}$. The observation of the spin Hall effect in unstrained n-GaAs, in which the k-linear effective field is small ${ }^{15}$, suggests that the extrinsic effect is dominant in that system ${ }^{3}$.
However, theoretical work argues that the cubic Dresselhaus term in GaAs could produce a non-negligible intrinsic spin Hall effect ${ }^{16}$.

Measurements in (110) quantum wells (QWs) may help distinguish between the two proposed mechanisms by allowing one to isolate the contributions of the Dresselhaus and Bychkov-Rashba fields. In two-dimensional systems, quantum confinement modifies the Dresselhaus spin splitting ${ }^{17}$. For the (110) QW, the Dresselhaus field is oriented along the growth direction, whereas this field is in-plane in conventional (001) heterostructures. As the Dresselhaus and Bychkov-Rashba fields are mutually perpendicular, one can tune the in-plane $B_{\text {int }}$ with the Bychkov-Rashba effect and the out-of-plane $B_{\text {int }}$ with the Dresselhaus field using engineered (110) heterostructures. In addition, two-dimensional systems provide a flexible architecture where carrier density, mobility and structural inversion asymmetry can be controlled using electric fields ${ }^{18}$.

Modulation-doped, digitally grown single QWs are grown by molecular beam epitaxy on (110) semi-insulating GaAs substrates. The QW structure behaves like a single $75 \AA \mathrm{Al}_{0.1} \mathrm{Ga}_{0.9}$ As QW with $\mathrm{Al}_{0.4} \mathrm{Ga}_{0.6}$ As barriers at a temperature $T=30 \mathrm{~K}$. For the optical measurements, a mesa is defined using a chemical etch (Fig. 1a) and contacts are made using annealed $\mathrm{AuGe} / \mathrm{Ni}$.

The spin polarization in the two-dimensional electron gas (2DEG) is spatially resolved using low-temperature scanning Kerr rotation microscopy ${ }^{19}$ in the Voigt geometry. A linearly polarized beam is tuned to the absorption edge of the QW (wavelength $\lambda=719 \mathrm{~nm}$ ) and directed normal to the sample through an objective lens, providing $\sim 1.1 \mu \mathrm{m}$ lateral spatial resolution. The rotation of the polarization axis of the reflected beam provides a measure of the electron spin polarization along the beam direction. A square wave voltage with maximum amplitude $\pm V_{\mathrm{p}}$ and frequency $511 \mathrm{~Hz}$ is applied to the device for lock-in detection. Measurements are performed in devices with electric fields applied along four different crystal directions in order to create a directional map of the internal fields. All of the data presented are measured at $T=30 \mathrm{~K}$ and we take $x=0 \mu \mathrm{m}$ to be the centre of the channel.

In Fig. 1b, we present Kerr rotation data as a function of the applied in-plane magnetic field $B_{\text {ext }}$ for positions near the two opposite edges of a channel aligned along the [001] direction. 

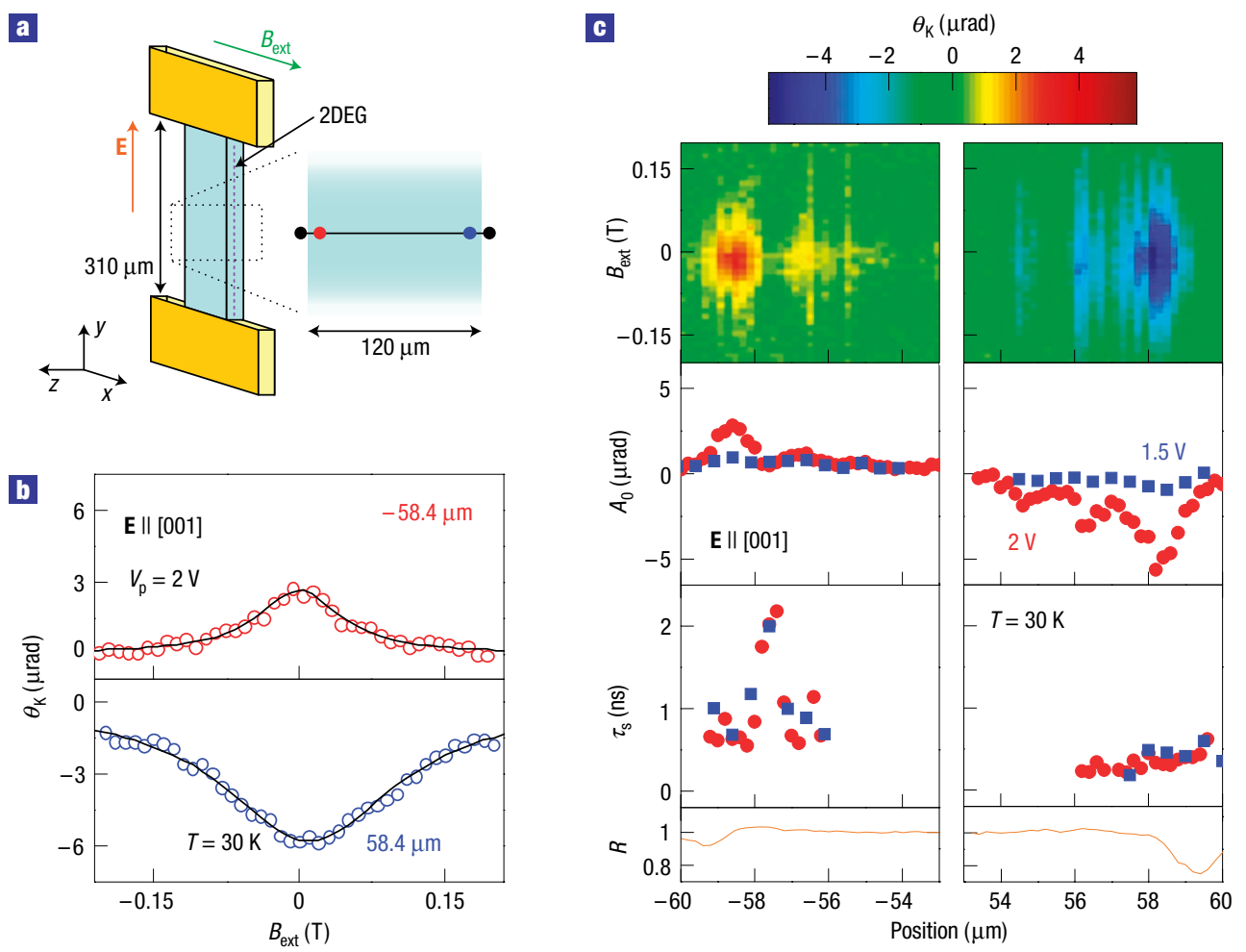

Figure 1 The spin Hall effect in a 2DEG. a, The device schematic and measurement geometry. The light-blue region indicates the mesa and the yellow regions are the contacts. $\mathbf{b}$, Kerr rotation (open circles) and fits (curves) as a function of applied in-plane magnetic field $B_{\text {ext }}$ for $x=-58.4 \mu \mathrm{m}$ (top, in red) and $x=+58.4 \mu \mathrm{m}$ (bottom, in blue). The channel has width $w=120 \mu \mathrm{m}$, length $I=310 \mu \mathrm{m}$ and mesa height $h=0.1 \mu \mathrm{m}$. A linear background is subtracted for clarity. c, $B_{\text {ext }}$ scans as a function of position near the edges of the channel of a device fabricated along the [001] direction for $V_{\mathrm{p}}=2 \mathrm{~V}$. Amplitude $A_{0}$, spin coherence time $\tau_{\mathrm{s}}$ and reflectivity $R$ are plotted for $V_{p}=1.5 \mathrm{~V}$ (blue squares) and $2 \mathrm{~V}$ (red circles).

These data correspond to a measurement of the Hanle effect using Kerr rotation ${ }^{20}\left(\theta_{\mathrm{K}}\right)$ and indicate the presence of an out-of-plane spin polarization when the data can be fitted to a lorentzian $A_{0} /\left[\left(\omega_{\mathrm{L}} \tau_{\mathrm{s}}\right)^{2}+1\right]$, where $A_{0}$ is peak Kerr rotation, $\omega_{\mathrm{L}}=g \mu_{\mathrm{B}} B_{\text {ext }} / \hbar$ is the Larmor precession frequency, $\tau_{\mathrm{s}}$ is the electron spin coherence time, $g$ is the electron $g$ factor, $\mu_{\mathrm{B}}$ is the Bohr magneton and $\hbar$ is the Planck constant. $A_{0}$ is of opposite sign for the two edges of the sample, which is a signature of the spin Hall effect.

In Fig. 1c, a one-dimensional spatial profile of the spin accumulation near the edges is mapped out by repeating $B_{\text {ext }}$ scans as a function of position. There are two spin Hall peaks at each edge, one around $x= \pm 58.6 \mu \mathrm{m}$ and one of smaller amplitude around $x= \pm 56.4 \mu \mathrm{m}$. This structure was not observed in measurements on bulk epilayers ${ }^{7}$ and could be due to an additional contribution from spin-polarized carriers undergoing spin precession about the in-plane Bychkov-Rashba field as they diffuse towards the centre of the channel. However, the asymmetry in $\left|A_{0}\right|$ for the right and left edges and a spatial dependence of $\tau_{\mathrm{s}}$ was also observed in previous measurements ${ }^{7}$. The reflectivity $R$ shows the position of the edges of the channel, at $x= \pm 59.4 \mu \mathrm{m}$.

In the [001]-oriented device, electrically induced spin polarization is observed only at the edges of the channel. In

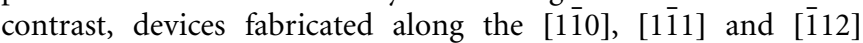
directions also show spin polarization at the centre of the channel. Figure $2 \mathrm{~b}$ shows data taken at $x=0 \mu \mathrm{m}$ for electric field $\mathbf{E}$

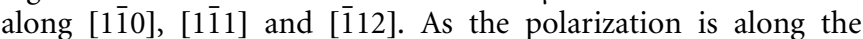
growth direction and depends on the direction of $\mathbf{E}$ relative to the crystal axes, we attribute this effect to the Dresselhaus field. The application of an electric field results in a non-zero average drift velocity of the electrons, which produces a non-zero effective magnetic field that orients spins $s^{5,6}$. Although the opposite sign of $A_{0}$ for $\mathbf{E} \|[1 \overline{1} 0]$ and $\mathbf{E} \|[1 \overline{1} 1]$ may seem surprising as these directions are only separated by $35.3^{\circ}$ in the (110) plane (Fig. 2a), it is consistent with the calculated $B_{\text {int }}$ due to the cubic Dresselhaus field in a (110) QW. This theory also predicts that $B_{\text {int }}$ should be zero for E $\|$ [001] (ref. 21), as observed.

Figure $2 \mathrm{c}$ shows a spatial profile of the spin polarization near the edges for a device aligned along [1 $1 \overline{1} 0] . A_{0}$ is negative across the entire channel, and $\left|A_{0}\right|$ increases with increasing voltage. From $-52 \mu \mathrm{m}<x<+52 \mu \mathrm{m},\left|A_{0}\right|$ is nearly constant across the channel. However, $\left|A_{0}\right|$ becomes smaller near the left edge of the channel, and a negative peak in $A_{0}$ is evident near the right edge, which is due to the spin Hall effect. The data for $V_{\mathrm{p}}=3 \mathrm{~V}$ suggest that there may be two spin Hall peaks, at $x=55.5$ and $57.5 \mu \mathrm{m}$, similar to the two peaks with $\sim 2 \mu \mathrm{m}$ spacing observed in the [001] device. We also observe that $A_{0}$ increases more dramatically with voltage for the spin Hall peak near the right edge than for the current-induced spin polarization across the rest of the channel.

We continue examining the direction dependence of the current-induced spin polarization with spatial scans of a channel aligned along [1iㅣ]. Figure 3 shows the spatial profile of the spin polarization near the edges of the channel. $A_{0}$ is positive across the entire channel, and $\left|A_{0}\right|$ is nearly constant from $-26 \mu \mathrm{m}<$ $x<+26 \mu \mathrm{m}$. However, there is a small positive peak around $x=-31 \mu \mathrm{m}$ and $\left|A_{0}\right|$ diminishes near the right edge of the channel.

We also perform spatially resolved measurements of a device aligned along [1112] (Supplementary Information, Fig. S1). Again, we observe a uniform spin polarization in the centre of the channel 

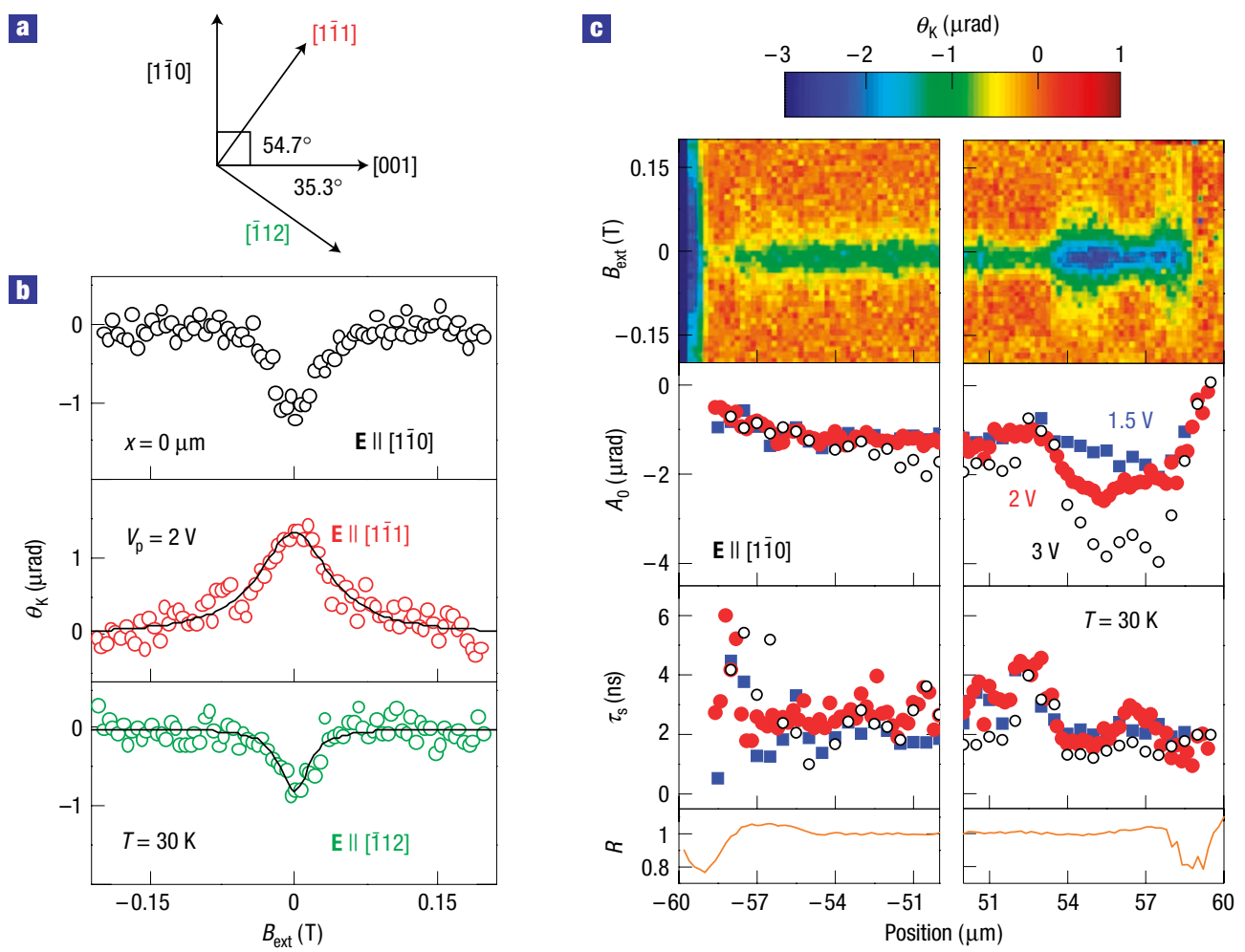

Figure 2 Current-induced spin polarization in a 2DEG. a, Relative orientations of crystal directions in the (110) plane. b, Kerr rotation (open circles) and fits (lines) as a function of $B_{\text {ext }}$ for $\mathbf{E} \|$ [110] (black), $\mathbf{E} \|[111]$ (red) and $\mathbf{E} \|\left[\overline{112}\right.$ (green) at the centre of the channel. $\mathbf{c}, B_{\text {ext }}$ scans as a function of position near the edges of the channel of a device fabricated along [110] with $W=118 \mu \mathrm{m}$ and $I=310 \mu \mathrm{m}$ for $V_{\mathrm{p}}=2 \mathrm{~V}$. Amplitude $A_{0}$, spin-coherence time $\tau_{\mathrm{s}}$ and reflectivity $R$ are plotted for $V_{\mathrm{p}}=1.5 \mathrm{~V}$ (blue filled squares), $2 \mathrm{~V}$ (red filled circles) and $3 \mathrm{~V}$ (black open circles).

and spin accumulation owing to the spin Hall effect at the edges of the channel. From our measurements on all four devices, we conclude that the spin Hall effect shows the same polarity for electric fields applied along all four crystal directions.

In Fig. 4, we present voltage dependences of $A_{0}$ and $\tau_{\mathrm{s}}$ for the spin Hall peaks in the [001] device and the current-induced spin polarization in the [110], [11 1$]$ and [1 12] devices. In Fig. 4a, we plot $A_{0}$ for the spin Hall peaks near the edges of the [001] channel and observe that $\left|A_{0}\right|$ increases with increasing $V_{\mathrm{p}}$. The nonlinearity of the increase in $\left|A_{0}\right|$ could be due to changes in the spin Hall profile or in the electrical response of the device. In contrast, we observe in Fig. $4 \mathrm{~b}$ that $\tau_{\mathrm{s}}=545 \pm 176 \mathrm{ps}$ and it does not have a clear voltage dependence over this range.

In order to explore the direction dependence of the current-induced spin polarization, we measure $A_{0}$ at $x=0 \mu \mathrm{m}$ for devices aligned along [11̄0], [1111] and [i112] as a function of $V_{\mathrm{p}}$, which we plot in Fig. 4c. We observe that the amplitude of the current-induced spin polarization increases with increasing $V_{\mathrm{p}}$, as expected. In addition, $\tau_{\mathrm{s}}=1,344 \pm 404 \mathrm{ps}$ and does not show a clear dependence on voltage (Fig. $4 \mathrm{~d}$ ). The direction dependence of $A_{0}$ reflects the strong $\mathbf{k}$ dependence of the Dresselhaus field.

In order to determine the mechanism of the spin Hall effect, we quantify the Rashba coefficient $\alpha$ by measuring the in-plane $B_{\text {int }}$ for our sample. The Bychkov-Rashba field has magnitude $\left|B_{\text {int }}\right|=\alpha|\mathbf{k}| / g \mu_{\mathrm{B}}$ and is oriented perpendicular to $\mathbf{k}$. $B_{\text {int }}$ can be observed as a shift in a Hanle ${ }^{22}$ or field-dependent Kerr rotation curve ${ }^{15}$ when we apply a d.c. voltage $V_{\text {d.c. }}$ along the [001] direction. Spins are injected optically into the QW and measured as a function of $B_{\text {ext }}$ after a time delay of $6 \mathrm{~ns}$. Figure 5 a shows Kerr rotation as a function of $B_{\text {ext }}$ for $V_{\text {d.c. }}=-2$ and $+2 \mathrm{~V}$. Lorentzian fits determine

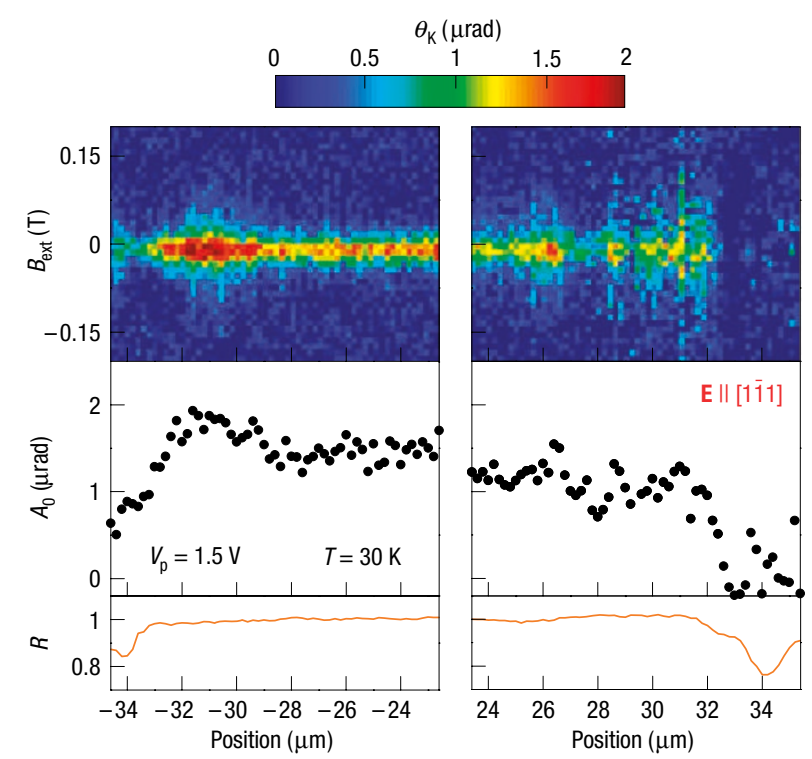

Figure 3 Spin polarization near the edges of a channel oriented along [1111]. $B_{\text {ext }}$ scans as a function of position near the edges of the channel of a device fabricated along [1 111$]$ with $w=68 \mu \mathrm{m}$ and $I=306 \mu \mathrm{m}$ for $V_{p}=1.5 \mathrm{~V}$. Amplitude $A_{0}$ and reflectivity $R$ are also plotted.

the centre of the peak, which is $-B_{\text {int }}$. In Fig. $5 \mathrm{~b}, B_{\text {int }}$ as a function of $V_{\text {d.c. }}$ can be fitted to a line with slope $1.77 \mathrm{mT} \mathrm{V}^{-1}$ and we 

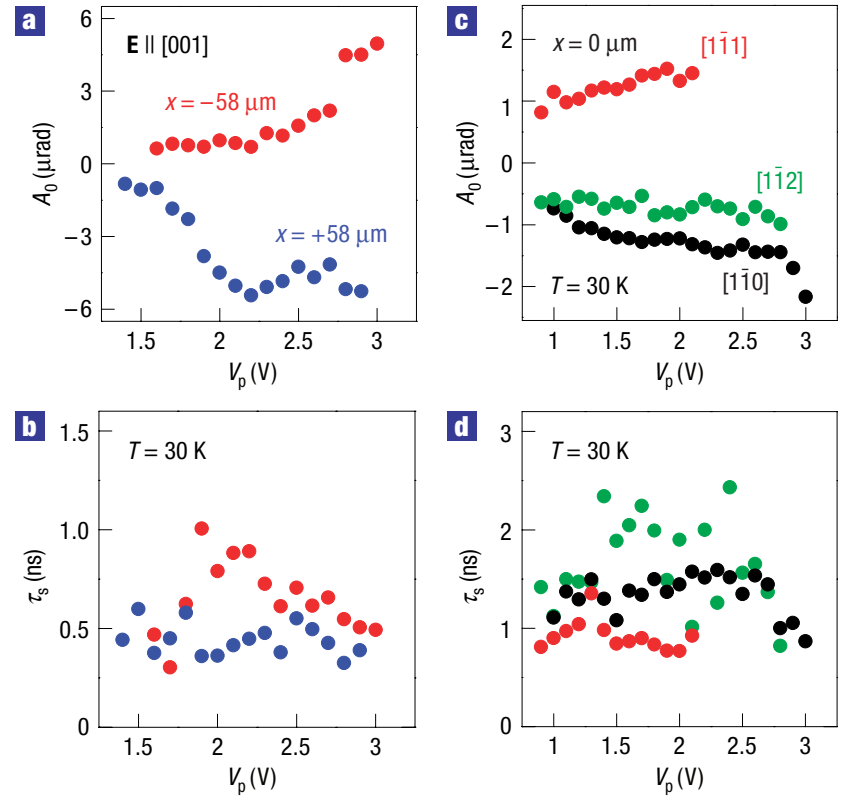

Figure 4 Voltage dependence of the electrically induced spin polarization.

a,b, Amplitude $A_{0}$ (a) and spin-coherence time $\tau_{\mathrm{s}}$ (b) of the spin Hall polarization as a function of voltage for $x=-58 \mu \mathrm{m}$ (red) and $x=+58 \mu \mathrm{m}$ (blue) for a device fabricated along [001]. c,d, $A_{0}(\mathbf{c})$ and $\tau_{\mathrm{s}}$ (d) of the current-induced spin polarization as a function of voltage $V_{p}$ for electric fields applied along [110] (black), [1111] (red) and [112] (green) measured at the centre of the channel $(x=0 \mu \mathrm{m})$.
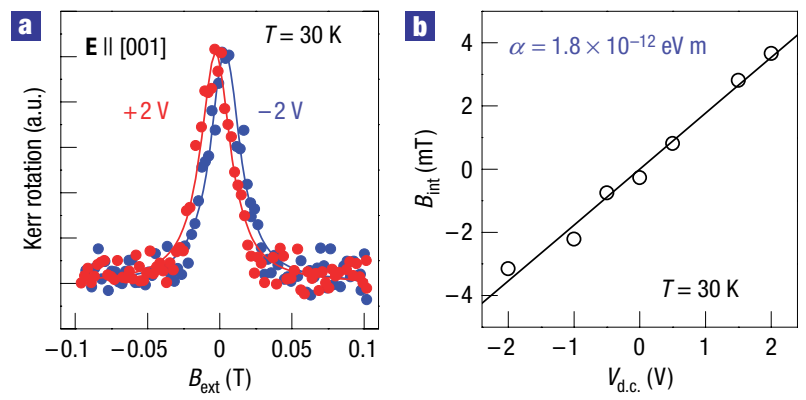

Figure 5 Measurement of the Bychkov-Rashba spin splitting. a, Kerr rotation as a function of $B_{\text {ext }}$ for $V_{\text {d.c. }}=-2 \mathrm{~V}$ (blue) and $V_{\text {d.c. }}=+2 \mathrm{~V}$ (red). The data were taken with a laser spot size of $30 \mu \mathrm{m}$. Lines are lorentzian fits. $\mathbf{b}$, In-plane effective magnetic field $B_{\text {int }}$ as a function of $V_{\text {d.c. }}$.

determine $\alpha=1.8 \times 10^{-12} \mathrm{eV} \mathrm{m}$. This small value for $\alpha$ is reasonable because this QW was designed to be symmetric, as $\alpha$ is a measure of the structural inversion asymmetry. This is also consistent with the observation that the current-induced spin polarization does not change significantly for the $\mathbf{E} \| B_{\text {ext }}$ geometry, where one would also measure spins that are oriented in-plane ${ }^{5}$. In addition, this value for $B_{\text {int }}$ yields a spatial spin precession period ${ }^{23}$ of $3.5 \mu \mathrm{m}$, which is similar to the $\sim 2 \mu \mathrm{m}$ distance observed between the spin Hall peaks in the [001] and [1 10 ] devices and suggests that the spacing between the spin Hall peaks could be due to spin precession. This relation could be confirmed by tuning $\alpha$ with a gate voltage ${ }^{18}$. Calculations of the intrinsic spin Hall effect for Rashba spin-orbit coupling show that the spin Hall conductivity should be non-zero when the Rashba splitting is larger than the disorder broadening ${ }^{12}$.
The ratio $\Delta_{0} \tau_{\mathrm{p}} / \hbar \sim 10^{-2}$, where $\Delta_{0}$ is the spin-splitting energy and $\tau_{\mathrm{p}}$ is the mean scattering time, and this ratio relates the strength of the spin-orbit coupling with impurity scattering ${ }^{24}$. In addition, the Dresselhaus terms are oriented out-of-plane in our sample and should not contribute to the spin Hall conductivity. Therefore, our data suggest that the spin Hall effect that we observe is dominated by the extrinsic spin Hall mechanism.

Spin-orbit engineering in two-dimensional systems allows for the manipulation of the magnitude and direction of the internal fields for sourcing spin polarization in non-magnetic semiconductors. Moreover, these interactions can be used to operate on electron spins by changing the direction of current, thereby enabling new degrees of control for quantum confined spintronic devices.

\section{METHODS}

\section{SAMPLE GROWTH AND DEVICE PREPARATION}

Conditions for the (110) growth are similar to those described in ref. 25; the substrate temperature is $490{ }^{\circ} \mathrm{C}$, the $\mathrm{As}_{4}$ beam equivalent pressure is $1.6 \times 10^{-5}$ torr and the growth rate of GaAs is $\sim 0.5$ monolayers $\mathrm{s}^{-1}$. The samples consist of four $14 \AA$ GaAs layers with $\mathrm{Al}_{0.4} \mathrm{Ga}_{0.6}$ As barriers separated by $6 \AA \mathrm{Al}_{0.4} \mathrm{Ga}_{0.6}$ As spacers. The barriers are delta-doped with silicon at $200 \AA$ from the QW structure on both the surface and the substrate side, with doping densities of $1.4 \times 10^{12}$ and $5.6 \times 10^{11} \mathrm{~cm}^{-2}$, respectively. In addition, silicon doping at $1 \times 10^{18} \mathrm{~cm}^{-3}$ is present within the $\mathrm{QW}$ region. Conventional Hall measurements at $T=5 \mathrm{~K}$ determine the sheet density $n_{\mathrm{s}}=1.9 \times 10^{12} \mathrm{~cm}^{-2}$ and mobility $\mu=940 \mathrm{~cm}^{2} \mathrm{~V}^{-1} \mathrm{~s}^{-1}$. Devices are aligned to the natural cleaves along [001] and [1111] such that an electric field $\mathbf{E}$ can be applied along the in-plane directions [001], [11 10$]$, [11 11$]$ and [i112]. Using time-resolved Kerr rotation $^{26}$, we determine $|g|=0.33$ for this sample and $\tau_{\mathrm{s}}=766 \mathrm{ps}$ at $B_{\text {ext }}=0.2 \mathrm{~T}$. The longitudinal spin coherence time is $3,250 \mathrm{ps}$ at $B_{\text {ext }}=0 \mathrm{~T}$. The relatively long spin-coherence times observed in (110) QWs ${ }^{27}$ compared with (001) $2 \mathrm{DEGs}{ }^{28}$ is due to the suppression of the D'yakonov-Perel' spin relaxation mechanism ${ }^{29}$. The data presented in this paper are from devices processed from one sample, but measurements performed on devices fabricated from a second sample verify the reproducibility of our results.

\section{MEASUREMENT OF BYCHKOV-RASHBA FIELD}

The shift in field-dependent Kerr rotation is used to measure the in-plane $B_{\text {int }}$ as a function of applied voltage in order to determine $\alpha$. As the contact resistance is large compared with the resistance of the channel, we consider the voltage drop across the channel $V_{\mathrm{c}}=\left(R_{\mathrm{c}} / R_{\mathrm{T}}\right) V_{\text {d.c. }}$, where $R_{\mathrm{c}}=980 \Omega$ is the resistance of the channel and $R_{\mathrm{T}}=10.3 \mathrm{k} \Omega$ is the total resistance of the device. As $\langle\mathbf{k}\rangle=\mu V_{\mathrm{c}} m_{\mathrm{e}}^{*} / \hbar l$, where the in-plane effective mass $m_{\mathrm{e}}^{*}=0.074 m_{\mathrm{e}}$ from a 14-band $\mathbf{K} \cdot \mathbf{p}$ calculation, and the spin-splitting energy $\Delta_{0}=g \mu_{\mathrm{B}} B_{\mathrm{int}}$, we determine $\alpha=1.8 \times 10^{-12} \mathrm{eV} \mathrm{m}$.

\section{Received 1 May 2005; accepted 24 June 2005; published 29 September 2005}

\section{References}

1. Wolf, S. A. et al. Spintronics: a spin-based electronics vision for the future. Science 294, $1488-1495(2001)$

2. Awschalom, D. D., Loss, D. \& Samarth, N. (eds) Semiconductor Spintronics and Quantum Computation (Springer, Berlin, 2002).

3. D'yakonov, M. I. \& Perel', V. I. Possibility of orienting electron spins with current. JETP Lett. 13, $467-469$ (1971)

4. Hirsch, J. E. Spin Hall effect. Phys. Rev. Lett. 83, 1834-1837 (1999).

5. Edelstein, V. M. Spin polarization of conduction electrons induced by electric current in two-dimensional asymmetric electron systems. Solid State Commun. 73, 233-235 (1990).

6. Aronov, A. G. \& Lyanda-Geller, Y. B. Nuclear electric resonance and orientation of carrier spins by an electric field. JETP Lett. 50, 431-434 (1989).

7. Kato, Y. K., Myers, R. C., Gossard, A. C. \& Awschalom, D. D. Observation of the spin Hall effect in semiconductors. Science 306, 1910-1913 (2004)

8. Wunderlich, J., Kaestner, B., Sinova, J. \& Jungwirth, T. Experimental observation of the spin-Hall effect in a two-dimensional spin-orbit coupled semiconductor system. Phys. Rev. Lett. 94, 047204 (2005).

9. Kato, Y. K., Myers, R. C., Gossard, A. C. \& Awschalom, D. D. Current-induced spin polarization in strained semiconductors. Phys. Rev. Lett. 93, 176601 (2004).

10. Silov, A. Yu. et al. Current-induced spin polarization at a single heterojunction. Appl. Phys. Lett. 85, 5929-5931 (2004).

11. Murakami, S., Nagaosa, N. \& Zhang, S. C. Dissipationless quantum spin current at room temperature. Science 301, 1348-1351 (2003).

12. Sinova, J. et al. Universal intrinsic spin Hall effect. Phys. Rev. Lett. 92, 126603 (2004)

13. Dresselhaus, G. Spin-orbit coupling effects in zinc blende structures. Phys. Rev. 100, $580-586$ (1955). 
14. Bychkov, Y. A. \& Rashba, E. I. Oscillatory effects and the magnetic susceptibility of carriers in inversion layers. J. Phys. C 17, 6039-6045 (1984).

15. Kato, Y. K., Myers, R. C., Gossard, A. C. \& Awschalom, D. D. Coherent spin manipulation without magnetic fields in strained semiconductors. Nature 427, 50-53 (2004)

16. Bernevig, B. A. \& Zhang, S. C. Spin splitting and spin current in strained bulk semiconductors. cond-mat/0412550 (2004)

17. D'yakonov, M. I. \& Kachorovskii, V. Yu. Spin relaxation of two-dimensional electrons in noncentrosymmetric semiconductors. Sov. Phys. Semicond. 20, 110-112 (1986).

18. Nitta, J., Akazaki, T., Takayanagi, H. \& Enoki, T. Gate control of spin-orbit interaction in an inverted $\mathrm{In}_{053} \mathrm{Ga}_{0.47} \mathrm{As} / \mathrm{In}_{0.52} \mathrm{Al}_{048}$ As heterostructure. Phys. Rev. Lett. 78, 1335-1338 (1997).

19. Stephens, J. et al. Spatial imaging of magnetically patterned nuclear spins in GaAs. Phys. Rev. B 68, 041307 (2003)

20. Stephens, J. et al. Spin accumulation in forward-biased MnAs/GaAs Schottky diodes. Phys. Rev. Lett. 93, 097602 (2004)

21. Winkler, R. Spin orientation and spin precession in inversion-asymmetric quasi-two-dimensional electron systems. Phys. Rev. B 69, 045317 (2004).

22. Kalevich, V. K. \& Korenev, V. L. Effect of electric field on the optical orientation of 2D-electrons. JETP Lett. 52, 230-235 (1990).

23. Kato, Y. K., Myers, R. C., Gossard, A. C. \& Awschalom, D. D. Electrical initialization and manipulation of electron spins in an L-shaped strained n-InGaAs channel. Appl. Phys. Lett. 87, 022503 (2005).

24. Schliemann, J. \& Loss, D. Dissipation effects in spin-Hall transport of electrons and holes. Phys. Rev. $B$ 69, 165315 (2004).
25. Pfeiffer, L. et al. Formation of a high quality two-dimensional electron gas on cleaved GaAs. Appl. Phys. Lett. 56, 1697-1699 (1990)

26. Crooker, S. A., Awschalom, D. D., Baumberg, J. J., Flack, F. \& Samarth, N. Optical spin resonance and transverse spin relaxation in magnetic semiconductor quantum wells. Phys. Rev. B 56, 7574-7588 (1997).

27. Ohno, Y., Terauchi, R., Adachi, T., Matsukura, F. \& Ohno, H. Spin relaxation in GaAs(110) quantum wells. Phys. Rev. Lett. 83, 4196-4199 (1999).

28. Sih, V. et al. Control of electron-spin coherence using Landau level quantization in a two-dimensional electron gas. Phys. Rev. B 70, 161313 (2004).

29. D'yakonov, M. I. \& Perel', V. I. Spin orientation of electrons associated with interband absorption of light in semiconductors. Sov. Phys. JETP 33, 1053-1059 (1971).

\section{Acknowledgements}

We acknowledge support from ARO, DARPA, NSF and ONR.

Correspondence and requests for materials should be addressed to D.D.A.

Supplementary Information accompanies this paper on www.nature.com/naturephysics.

Competing financial interests

The authors declare that they have no competing financial interests.

Reprints and permission information is available online at http://npg.nature.com/reprintsandpermissions/ 